\title{
Evaluation of the Potential Use of Levan Polysaccharide in Paper Conservation
}

\author{
Rumeysa Ozen Saglam $\mathbb{D}^{1},{ }^{1}$ Seval Genc, ${ }^{2}$ and Ebru Toksoy Oner $\mathbb{D}^{1}$ \\ ${ }^{1}$ Industrial Biotechnology and Systems Biology Research Group (IBSB), Department of Bioengineering, Marmara University, \\ Istanbul, Turkey \\ ${ }^{2}$ Department of Metallurgical and Materials Engineering, Marmara University, Istanbul, Turkey
}

Correspondence should be addressed to Ebru Toksoy Oner; ebru.toksoy@marmara.edu.tr

Received 10 January 2020; Accepted 20 July 2020; Published 10 August 2020

Guest Editor: Hussein M. Mahmoud

Copyright (c) 2020 Rumeysa Ozen Saglam et al. This is an open access article distributed under the Creative Commons Attribution License, which permits unrestricted use, distribution, and reproduction in any medium, provided the original work is properly cited.

\begin{abstract}
Conservation of paper-based materials, namely, manuscripts, includes repair of tears, gap filling, consolidation, and fixation, which requires the use of adhesives. The adhesive in use for these purposes should be compatible with the chemical and physical properties of paper and should present good aging characteristics. In this study, we conducted a set of experiments with paper samples on which fructan-based biopolymer Halomonas levan (Hlevan) was applied for the first time and compared with glucan-based adhesive starch. Adhesive-applied samples were initially prepared by the application of different historical recipes of sizing and ink. Then, they underwent accelerated thermal aging. Chemical and physical changes of paper samples (crystallinity, $\mathrm{pH}$, and color) were analyzed. Finally, Principal Component Analysis was performed to identify dominating factors affecting sample behavior with applied adhesives. This study demonstrates the aging characteristics of Hlevan on cellulose-based materials. The results showed that there is a color shift from blue to yellow for all samples after aging, but it is more pronounced for some samples with Hlevan. In addition, Hlevan is slightly less acidic than starch in all cases and acted as a plasticizer for a specific kind of sizing material. The methodology of this research is also efficacious in terms of understanding the interaction of paper media with the adhesives and their effects on aging.
\end{abstract}

\section{Introduction}

Levan is a fructose-based homopolysaccharide, a fructan that is mainly composed of $\beta$-D-fructofuranose residues linked by $\beta$-(2-6) glycosidic bonds (see Figure 1). It serves both structural and functional roles and stands out from other natural polymers by its unique combination of properties such as strong adhesivity, self-assembly into spherical colloids in water, very low intrinsic viscosity, high biocompatibility, and health benefits, which in turn make this unusual polysaccharide a valuable polymer for various applications in cosmetic, pharmaceutical, food, and other industries [1]. Whereas levan is also produced by a limited number of plant species at a low degree of polymerization (DP 10-100), longer levan chains (>DP $10^{3}-10^{4}$ ) offering a broader spectrum of applications are basically produced by miscellaneous microorganisms both extracellularly and at high titers from sucrose by the hydrolysis and transfructosylation action of levansucrase enzyme [2,3]. Besides mesophilic levan producers from a wide range of genera that include Acetobacter, Bacillus, Erwinia, Gluconobacter, Microbacterium, Pseudomonas, and Zymomonas, as well as many acetic acid and lactic acid bacteria, Halomonas cultures hold distinctive industrial potential due to their added advantages such as high yield and nonsterile production capacity under high salinity $[4,5]$.

The first extremophilic producer and novel species, Halomonas smyrnensis AAD6T, has been shown to produce levan at high titers [6], and subsequent studies by our research group revealed that levan produced by these cultures displays antioxidant, anticancer [7], and heparin mimetic [8] activities. Also, it could serve as a bioflocculating agent [9] and 


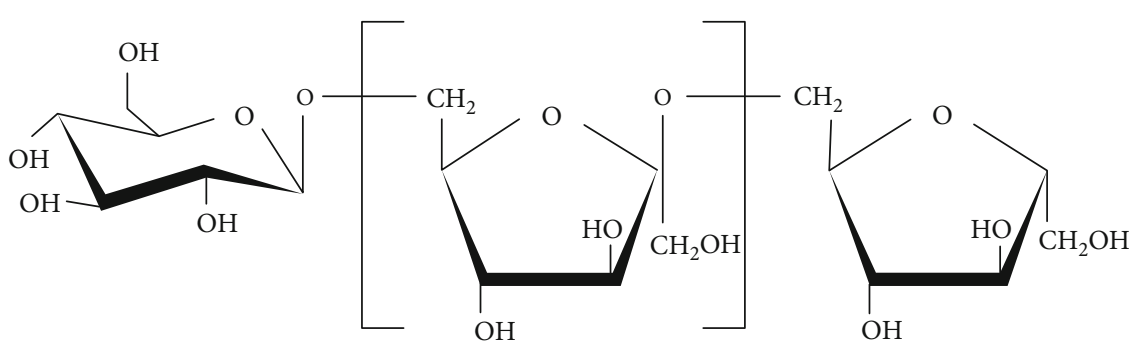

Figure 1: Chemical structure of levan.

functional biomaterial in many high-value applications such as drug carrier systems $[6,10,11]$, laser-deposited nanostructured bioactive surfaces, adhesive multilayer [12], blend [13] and free-standing films [14], and temperature-responsive hydrogels [15]. Despite the wide range use of levan as a biomaterial in several fields, its potential use for the conservation of cultural heritage artifacts has not been reported yet. As discussed by Combie and friends [16], levan has higher tensile and shear strength when compared to other natural polymers such as carboxymethylcellulose, corn starch, dextran, guar gum, xanthan gum, and sodium alginate. Its comparison with the starch paste by means of aging characteristics on cellulosic materials can be a practical method as the starch paste is generally the main adhesive of choice in paper conservation according to an international survey conducted by Alexopoulou and Zervos [17]. Although synthetic polymers are in a wide range of utilization in conservation, their application in paper conservation seemed to be limited [18]. In addition, when adhesives presently in use for paper conservation, namely, starch paste, unsupported ArchibondTM, carboxymethylcellulose, hydroxypropylcellulose, and methylcellulose, were analyzed for their chemical stability and fungal bioreceptivity, despite its high bioreceptivity, starch showed better aging characteristics among them [19]. Thus, any natural polymer showing superior or akin aging characteristics can pave the way for alternative use in this field. Adhesive properties like tensile strength, heat stability, and reversibility of levan have been revealed, but its long-term effects on paper which will reveal its potential of utilization for restoration or conservation process has not been studied yet [20].

When literature was investigated, it was revealed that starting from the third century, different sizing techniques had been used for coating the paper surface to make it easy to write on it. The materials used were natural substances such as gum, glue, starch, and egg [21]. Also, it is known that not only manuscripts but also drawings prevalently may include such materials. A conducted survey in British Museum's database in 2016 affirmed that more than 50\% of drawings were in "brown ink," i.e., iron gall ink, between the fifteenth and seventieth century. It is also acknowledged that iron gall ink resulted in brittleness on paper over time and color shift from black to brown in drawings [22]. Another ink used in conservation is called "carbon black" which has problems of penetrating into paper thus results in less resistance when compared with iron gall ink.

Reports on studies where different kinds of inks and sizing materials had been used generally point to a wide range of problems. Conservation of paper-based materials includes repair of tears, gap filling, consolidation, and fixation, which requires the use of adhesives. To be used in conservation treatments, these materials need to meet some qualifications like being chemically durable in integration with paper substrate $[19,23]$. The aging of paper is accordingly investigated using some parameters like crystallinity, $\mathrm{pH}$, and color change, which are also affected by the chemical composition of materials present on paper like sizing, painting, or ink. So, when investigating the aging characteristics of a material and its effects on paper, it is important to include such variables to imitate real substrates. To realize this, we conducted a set of experiments analyzing chemical changes of paper which were prepared by the application of different formulations of sizing, ink, and adhesives, namely, levan by Halomonas smyrnensis, Halomonas levan (HLevan), and starch. Samples underwent thermal aging so that the long-term effects of adhesives can be compared. Finally, Principal Component Analysis was performed to identify dominating factors affecting sample behavior with applied adhesives.

\section{Materials and Methods}

\subsection{Sample Preparation}

2.1.1. Substrates. Grade 1 Whatman filter paper was used as a model sample material in order to compare the compatibility of Hlevan in different proportions. The selection of filter paper was mainly due to the unbleached nature and neutral $\mathrm{pH}$ of the material in addition to its high cellulose content and lack of impurities, the presence of which might cause deviation from usual results [19].

2.1.2. Sizing Recipes. On historical papers, a process called sizing has been applied to prepare a paper surface for writing, illuminating, or painting. It is the process that prevents cellulose fiber from absorbing excess water by means of applying some natural substances on it. The surface of the sheet is covered with these substances by soaking it into sizing material or simply by brushing with them [21].

Out of the five historic sizing recipes, i.e., cucumber seed, egg white with fig milk, starch with alum, rice with gum Arabic, and egg white with alum, only two were chosen concerning both their easy applicability and wide range utilization on manuscripts [24-26].

Recipes that were assessed are listed below:

(1) Rice and Gum Arabic. Rice was boiled in water and smashed. The mixture was filtrated. $2 \mathrm{ml}$ of gum arabic was 


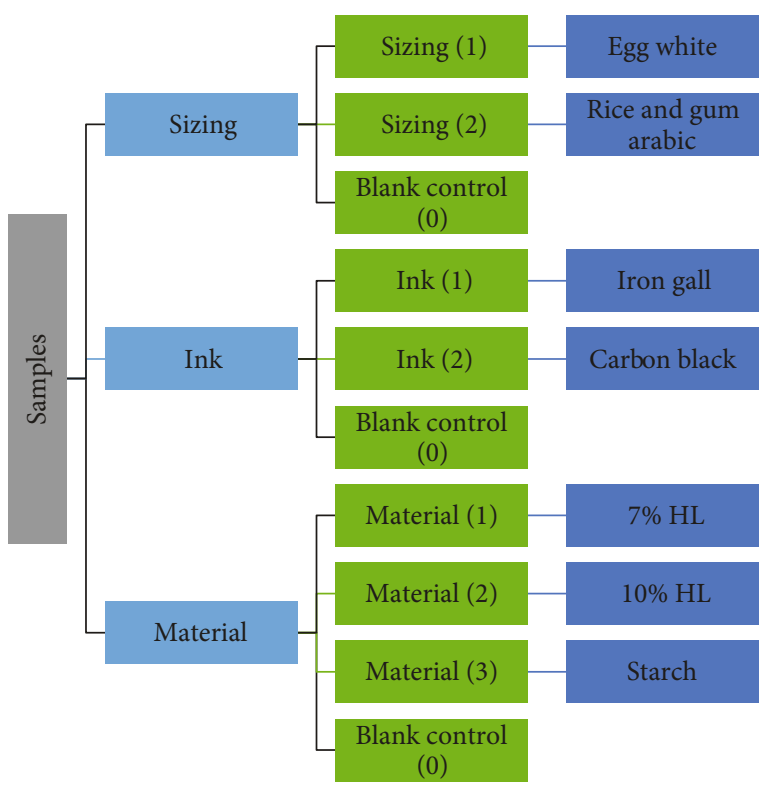

FIGURE 2: Schematic diagram for the preparation of paper samples.

added, and the solution was boiled again. After the solution cooled to room temperature, the paper was wiped with it [26].

(2) Egg White with Alum Sizing Preparation. $5 \mathrm{~g}$ of alum was added into the egg white and mixed continuously at room temperature for 20 minutes. The solution was filtered and rested for 2 hours at room temperature. Sample papers were wiped with it [26].

The selected recipes would enable us to investigate the interaction of both carbohydrate and protein-based sizing with testing biomaterial.

2.1.3. Ink Recipes. In historic manuscripts, mainly two types of ink were used. Therefore, both ink recipes were applied following sizing applications:

(1) Ink (1): Iron Gall. $2 \mathrm{~g}$ of gum arabic was dissolved in water and set aside. Two galls were crushed and covered with $75 \mathrm{ml}$ of water in a beaker where a brown colored solution is obtained after overnight incubation at room temperature. $2 \mathrm{~g}$ of iron sulfate was dissolved in $45 \mathrm{ml}$ of water and mixed with filtered gall and gum arabic solutions [27].

(2) Ink (2): Carbon Black. A pan lid was burned to get carbon black. Alcohol was added and waited to be evaporated. Gum arabic and water were added [28].

\subsubsection{Adhesive Preparation}

(1) Microbial Levan Production. Levan from H. smyrnensis was produced using a sucrose-based medium at $37^{\circ} \mathrm{C}$ in a stirred tank fermenter as described before [29]. Then, the medium was centrifuged for at least $10 \mathrm{~min}$ to remove cells. After centrifugation, levan in the supernatant phase was
TABLE 1: Variations in color coordinates of samples after aging.

\begin{tabular}{|c|c|c|c|c|c|}
\hline Group codes & Sample codes & $\Delta E$ & $\Delta L$ & $\Delta a$ & $\Delta b$ \\
\hline 0.0 & 0.0 .0 & 0.827164 & -0.52 & -0.13 & 0.63 \\
\hline 0.0 & 0.0 .1 & 1.533004 & 1.49 & 0.34 & 0.12 \\
\hline 0.0 & 0.0 .2 & 2.35083 & -0.6 & 2 & 1.08 \\
\hline 0.0 & 0.0 .3 & 4.393609 & 0.79 & -1.06 & 4.19 \\
\hline 0.1 & 0.1 .0 & 17.30719 & -1.28 & 2.03 & 17.14 \\
\hline 0.1 & 0.1 .1 & 14.10067 & -0.89 & 2.32 & 13.88 \\
\hline 0.1 & 0.1 .2 & 13.97278 & -4.52 & 2.41 & 13 \\
\hline 0.1 & 0.1 .3 & 13.75846 & 0.54 & 1.26 & 13.69 \\
\hline 0.2 & 0.2 .0 & 7.839043 & -7.59 & 0.03 & 1.96 \\
\hline 0.2 & 0.2 .1 & 9.029474 & -0.69 & 2.88 & 8.53 \\
\hline 0.2 & 0.2 .2 & 2.666702 & 0.81 & 0.06 & 2.54 \\
\hline 0.2 & 0.2 .3 & 3.154267 & -0.38 & -0.09 & 3.13 \\
\hline 1.0 & 1.0 .0 & 28.45094 & -9.33 & 4.37 & 26.52 \\
\hline 1.0 & 1.0 .1 & 52.20442 & -24.74 & 15.88 & 43.14 \\
\hline 1.0 & 1.0 .2 & 55.26563 & -29.88 & 18.32 & 42.73 \\
\hline 1.0 & 1.0 .3 & 39.34017 & -27.92 & 3.02 & 27.55 \\
\hline 1.1 & 1.1.0 & 17.06307 & -2.99 & 0.8 & 16.78 \\
\hline 1.1 & 1.1.1 & 15.42353 & -5.43 & 1.02 & 14.4 \\
\hline 1.1 & 1.1.2 & 15.88939 & -8.37 & 0.66 & 13.49 \\
\hline 1.1 & 1.1.3 & 12.05018 & -3.52 & -0.58 & 11.51 \\
\hline 1.2 & 1.2 .0 & 2.201681 & -2.17 & -0.19 & -0.32 \\
\hline 1.2 & 1.2 .1 & 4.153372 & -3.15 & 0.88 & 2.56 \\
\hline 1.2 & 1.2 .2 & 5.909357 & -1.84 & 3.75 & 4.18 \\
\hline 1.2 & 1.2 .3 & 4.386434 & -1.16 & 1.54 & 3.94 \\
\hline 2.0 & 2.0 .0 & 6.575546 & 0.59 & -1.19 & 6.44 \\
\hline 2.0 & 2.0 .1 & 7.073005 & 0.8 & -1.57 & 6.85 \\
\hline 2.0 & 2.0 .2 & 13.30259 & -0.39 & -2.1 & 13.13 \\
\hline 2.0 & 2.0 .3 & 7.036796 & 0.5 & -1.39 & 6.88 \\
\hline 2.1 & 2.1 .0 & 14.80861 & 3.37 & -0.04 & 14.42 \\
\hline 2.1 & 2.1.1 & 11.03857 & -2.24 & 0.79 & 10.78 \\
\hline 2.1 & 2.1 .2 & 9.176154 & -4.29 & 0.16 & 8.11 \\
\hline 2.1 & 2.1 .3 & 15.68494 & -2.46 & 2.36 & 15.31 \\
\hline 2.2 & 2.2 .0 & 7.925238 & 7.82 & -0.21 & 1.27 \\
\hline 2.2 & 2.2.1 & 2.327466 & -0.77 & 0.61 & 2.11 \\
\hline 2.2 & 2.2 .2 & 0.796492 & 0.4 & -0.3 & 0.62 \\
\hline 2.2 & 2.2 .3 & 1.544636 & 1.41 & -0.27 & 0.57 \\
\hline
\end{tabular}

recovered by ethanol addition. Retrieved levan pellets were redissolved in distilled water and purified with dialysis for 5 days. Following a final stage of air drying in a laboratory oven at $60^{\circ} \mathrm{C}$, levan samples were weighted and then used to prepare $7 \%$ and $10 \%(w / v)$ Hlevan solutions in distilled water.

(2) Starch. Starch (Sigma-Aldrich S9765) was used to prepare a starch solution in distilled water at $10 \%(w / v)$ concentration [30]. Starch was cooked after leaving for $30 \mathrm{~min}$ in distilled water with the same procedure as described by other authors [19]. We wanted to investigate two types of natural polymers, specifically Hlevan and starch by examining the effects on paper specimens before and after aging in order 

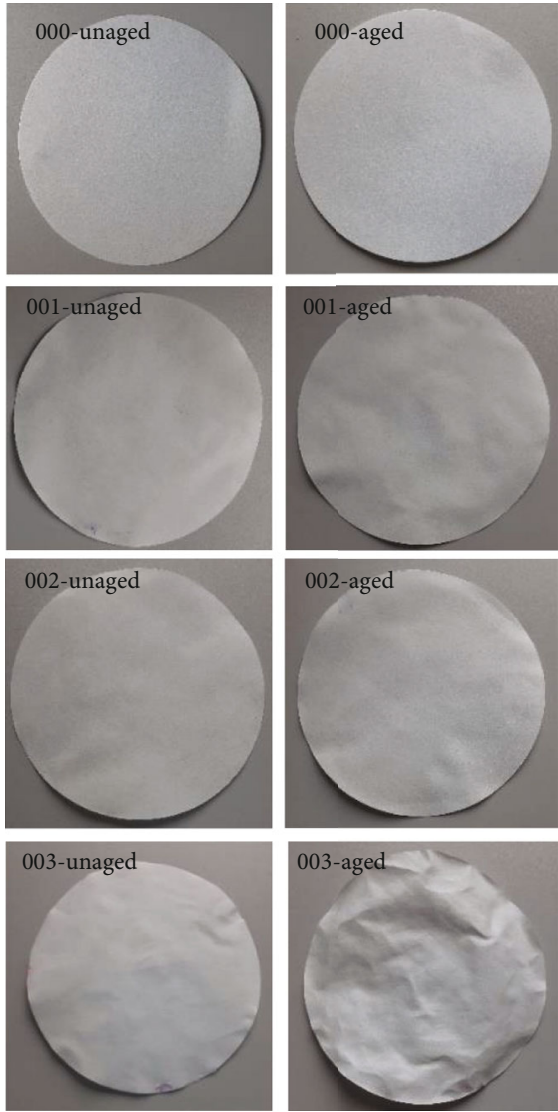

(a)
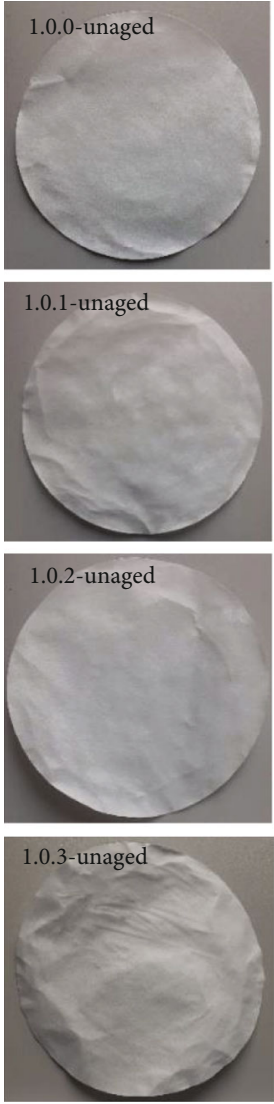

(b)
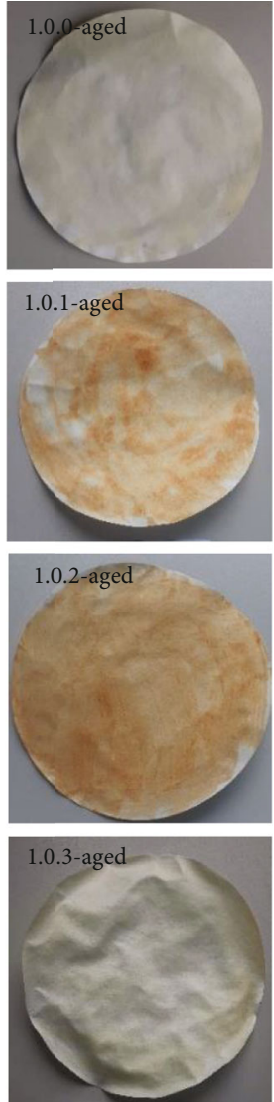
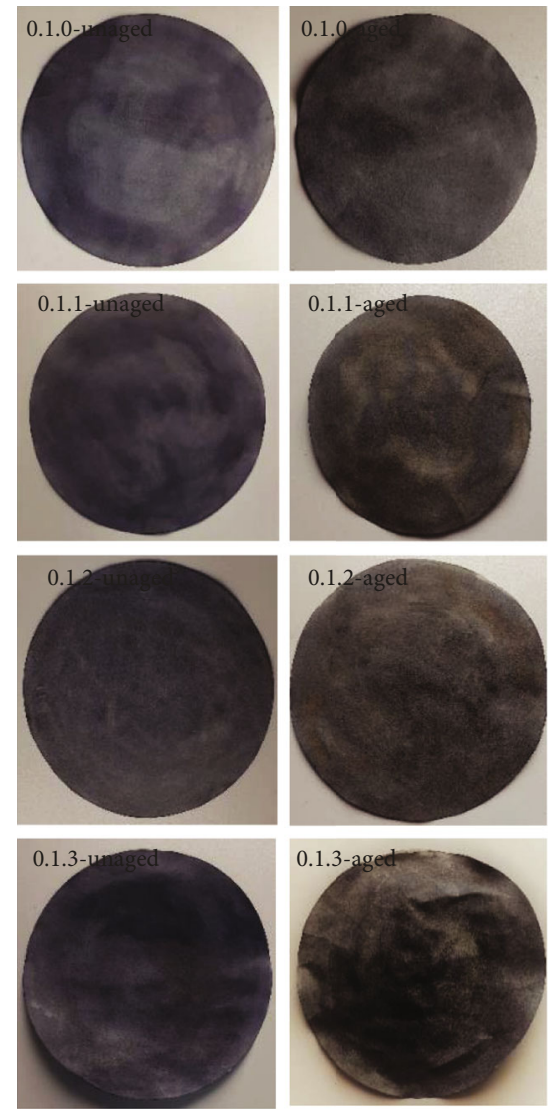

(c)

FIGURE 3: Before and after aging photos for the groups of samples that most color shift was observed: (a) group 0.0, (b) group 1.0, and (c) group 0.1 . Among $7 \%$ and $10 \%$ Hlevan-applied samples $(0.0 .1-0.0 .2 / 1.0 .1-1.0 .2 / 0.1 .1-0.1 .2)$, the ones that combined with egg-white sizing (1.0.1-1.0.2) showed distinguishable color shift.

to investigate the compatibility and potential of Hlevan. To realize this, along with control groups for each, 2 different sizing conditions, 2 types of inks, and 3 different formulations of adhesives $(3 \times 3 \times 4=36$ samples in total $)$ were used. Each paper sample combines one type of sizing, ink, and material including control groups (see Figure 2). Codes were given to samples to make tracking easier. For example, code 1.2.3 represents the combination for sizing 1 (egg white-alum), ink 2 (carbon black), and material 3 (starch), respectively. Making these combinations provided a clearer examination to see the effect of each variable on overall results. The groups and codes of samples were given in Supplement 1 .

2.2. Accelerated Aging Procedure. In the scope of this study, it is significant to be able to simulate a long-term degradation process. We preferred thermally accelerated aging to model the condition of historical papers and followed the conditions listed in the ISO 5630-4:1986 specification for paperbased materials. The prepared samples were hanged in a temperature-controlled oven and kept for 3 days at a temperature of $120^{\circ} \mathrm{C}$ [31].

2.3. Colorimetric Analysis. Color stability is another important variable to consider for the conservation process of paper artifacts. Many internal and external factors may cause a change in color. Thus, it is important to investigate the durability of color after the application of adhesives and aging. For colorimetric measurements, Datacolor Spectroflash SF 600 was used. CIE $L * a * b *$ coordinates were recorded before and after aging. $L *$ is the level of brightness; $a *$ corresponds to red-green coordinate while $b *$ is the measure of yellow-blue colors.

2.4. $\mathrm{pH}$ Test. The $\mathrm{pH}$ measurement is a simple yet very important parameter to identify the applicability of treatments during the restoration and conservation of artifacts [32]. Among different $\mathrm{pH}$ measurement techniques, the most accurate technique called the cold extraction method according to the standard TAPPI T 529 OM-2004 was used. $1 \mathrm{~g}$ of paper samples was left in $70 \mathrm{ml}$ of cold, distilled water between 1 and 20 hours. The $\mathrm{pH}$ measurements were carried out using a Mettler Toledo S400-B SevenExcellenceTM pH meter (Mettler Toledo, Switzerland).

2.5. XRD Analysis. The crystallinity index is a way of understanding the physical and chemical states of the paper. The flexibility of cellulose fiber which is related to its amorphous regions decreases over aging, making the paper more crystalline, accordingly more brittle [33]. X-ray diffraction measurements of samples before and after aging were carried 
out with Bruker D2 Phaser $\mathrm{CuK} \alpha$ radiation which was generated at $30.0 \mathrm{kV}$ and $10.0 \mathrm{~mA}$. The $\mathrm{CuK} \alpha$ radiation has a wavelength of $0.154184 \mathrm{~nm}$. Scans were obtained from 5 to $30^{\circ} 2 \theta$ in $0.01^{\circ}$ steps for 19.2 seconds per step. The peaks of diffractograms were analyzed using OriginPro 2020 version 9.7.0.188, and the crystalline index was calculated using the Segal method [34]:

$$
X_{c}=\frac{I_{002}-I_{\mathrm{am}}}{I_{002}} \times 100 \% \text {, }
$$

where $X_{c}$ expresses the relative degree of crystallinity, $I_{002}$ stands for the maximum intensity of lattice diffraction, and $I_{\mathrm{am}}$ is the intensity of the amorphous scatter at $2 \theta=18^{\circ}$ in arbitrary units [34-37].

\section{Results and Discussion}

3.1. Chromatic Changes. Variations of the chromatic coordinate $L * a * b *$ values after aging are given in Table 1 . Positively higher DE* values in CIELab Unit are the consequence of the drastic changes in $b *$ and $L *$ values which demonstrates a color shift from blue to yellow and decrease in lightness. Samples showing drastic color change after aging were mainly samples that were sized with egg white and alum recipe or dyed with iron gall ink. This color change was more noticeable for the samples with Hlevan than starch (see Figure 3).

The reaction between compounds maintaining free amino groups and carbonyl groups of reduced sugars results in color change, and this reaction is known as Maillard reaction. Here, egg white (or albumin) is a protein which reacts to form conjugated double bonds with the carbonyl group of carbohydrates. The resulted unstable N-substituted glycosylamine immediately undergoes further reaction to produce melanoidins (brown polymers). What is more, the Maillard reaction is accelerated in the presence of high temperature [38-41]. Although starch molecules have reducing ends, they also have a huge molecular weight to dilute down that reducing end to well below one in a thousand. Thus, unlike in Hlevan with lower molecular weight, detecting reducing ends above the noise level of the analytical method is difficult in the case of starch. Particularly, color shift to yellow and fading in samples with iron gall ink is due to the breaking down of ferric tannate complex into brown-yellow chemical products during aging [42]. Moreover, chain scission and dihydroxylation of cellulose also give rise to the development of free radicals that lead to yellowing. Extremely high $\Delta E$ and $\Delta b$ values may be because of the formation of a permanent cellulose-material complex, implying an increase in the rate of aging [43].

3.2. $p H$. The $\mathrm{pH}$ of the papers decreased in all cases after aging. Moreover, it decreases as the concentration of Hlevan increases. For example, samples with carbon black ink showed a change in $\mathrm{pH}$ from 6.72 to 5.90 and 6.21 to 5.85 for increasing Hlevan concentration. In the same manner, starch also caused a decrease in $\mathrm{pH}$ after aging. However, in all cases, starch resulted in more acidic media on paper
TABLE 2: Crystallinity index (CI) and $\mathrm{pH}$ of test specimens with sample and group codes before and after thermal aging.

\begin{tabular}{|c|c|c|c|c|c|}
\hline $\begin{array}{l}\text { Group } \\
\text { codes }\end{array}$ & $\begin{array}{l}\text { Sample } \\
\text { codes }\end{array}$ & $\begin{array}{l}\text { CI-before } \\
\text { aging }\end{array}$ & $\begin{array}{l}\text { CI-after } \\
\text { aging }\end{array}$ & $\begin{array}{l}\text { pH-before } \\
\text { aging }\end{array}$ & $\begin{array}{l}\mathrm{pH} \text {-after } \\
\text { aging }\end{array}$ \\
\hline 0.0 & 0.0 .0 & 0.87 & 0.88 & 6.02 & 5.97 \\
\hline 0.0 & 0.0 .1 & 0.86 & 0.87 & 6.12 & 5.80 \\
\hline 0.0 & 0.0 .2 & 0.85 & 0.87 & 6.06 & 5.12 \\
\hline 0.0 & 0.0 .3 & 0.85 & 0.84 & 5.79 & 4.98 \\
\hline 0.1 & 0.1 .0 & 0.82 & 0.80 & 3.24 & 3.15 \\
\hline 0.1 & 0.1 .1 & 0.79 & 0.83 & 3.32 & 3.34 \\
\hline 0.1 & 0.1 .2 & 0.81 & 0.84 & 3.13 & 3.09 \\
\hline 0.1 & 0.1 .3 & 0.81 & 0.81 & 3.02 & 3.00 \\
\hline 0.2 & 0.2 .0 & 0.84 & 0.84 & 6.75 & 5.29 \\
\hline 0.2 & 0.2 .1 & 0.83 & 0.84 & 6.72 & 5.90 \\
\hline 0.2 & 0.2 .2 & 0.82 & 0.84 & 6.21 & 5.85 \\
\hline 0.2 & 0.2 .3 & 0.83 & 0.83 & 6.25 & 5.72 \\
\hline 1.0 & 1.0 .0 & 0.84 & 0.86 & 4.25 & 4.12 \\
\hline 1.0 & 1.0 .1 & 0.84 & 0.85 & 4.11 & 4.02 \\
\hline 1.0 & 1.0 .2 & 0.83 & 0.82 & 4.13 & 4.03 \\
\hline 1.0 & 1.0 .3 & 0.80 & 0.85 & 4.25 & 4.17 \\
\hline 1.1 & 1.1 .0 & 0.85 & 0.85 & 3.46 & 3.43 \\
\hline 1.1 & 1.1.1 & 0.80 & 0.85 & 3.55 & 3.55 \\
\hline 1.1 & 1.1 .2 & 0.80 & 0.82 & 3.41 & 3.32 \\
\hline 1.1 & 1.1 .3 & 0.80 & 0.83 & 3.84 & 3.41 \\
\hline 1.2 & 1.2 .0 & 0.74 & 0.79 & 4.18 & 3.87 \\
\hline 1.2 & 1.2 .1 & 0.75 & 0.78 & 4.16 & 3.93 \\
\hline 1.2 & 1.2 .2 & 0.78 & 0.82 & 4.14 & 3.84 \\
\hline 1.2 & 1.2 .3 & 0.75 & 0.78 & 4.10 & 3.80 \\
\hline 2.0 & 2.0 .0 & 0.86 & 0.86 & 5.94 & 5.60 \\
\hline 2.0 & 2.0 .1 & 0.84 & 0.84 & 5.99 & 5.52 \\
\hline 2.0 & 2.0 .2 & 0.82 & 0.85 & 5.78 & 5.35 \\
\hline 2.0 & 2.0 .3 & 0.82 & 0.79 & 5.86 & 5.54 \\
\hline 2.1 & 2.1 .0 & 0.85 & 0.85 & 3.26 & 2.78 \\
\hline 2.1 & 2.1 .1 & 0.79 & 0.82 & 3.41 & 3.05 \\
\hline 2.1 & 2.1 .2 & 0.77 & 0.80 & 3.44 & 2.85 \\
\hline 2.1 & 2.1 .3 & 0.79 & 0.81 & 3.36 & 2.87 \\
\hline 2.2 & 2.2 .0 & 0.69 & 0.77 & 6.02 & 6.00 \\
\hline 2.2 & 2.2 .1 & 0.73 & 0.78 & 5.89 & 5.80 \\
\hline 2.2 & 2.2 .2 & 0.75 & 0.82 & 5.89 & 5.65 \\
\hline 2.2 & 2.2 .3 & 0.82 & 0.83 & 5.55 & 5.50 \\
\hline
\end{tabular}

support than Hlevan did (see Table 2). Samples with iron gall ink showed highly acidic nature, $\mathrm{pH}$ values ranging between 2 and 4, as expected while the $\mathrm{pH}$ of blank stayed almost neutral before and after aging. The results showed that starch yielded more acidity than Hlevan-applied samples as an adhesive. It is the most noteworthy result of $\mathrm{pH}$ measurements.

Also, samples including alum in their sizing became acidic. Although a decrease in $\mathrm{pH}$ upon aging was less for the samples with iron gall ink and/or egg white sized, it is important to note that their initial $\mathrm{pH}$ was already lower than 


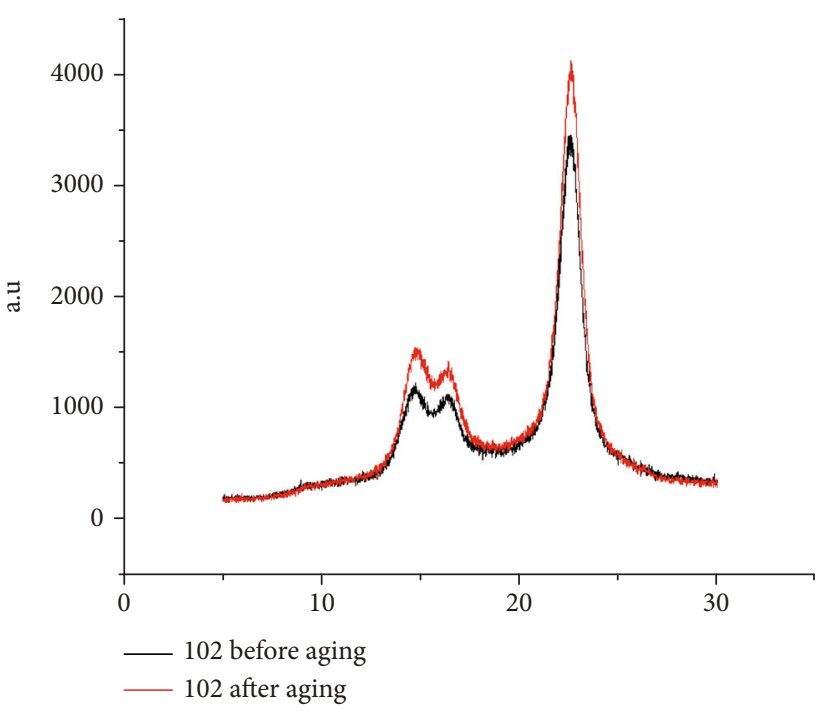

(a)

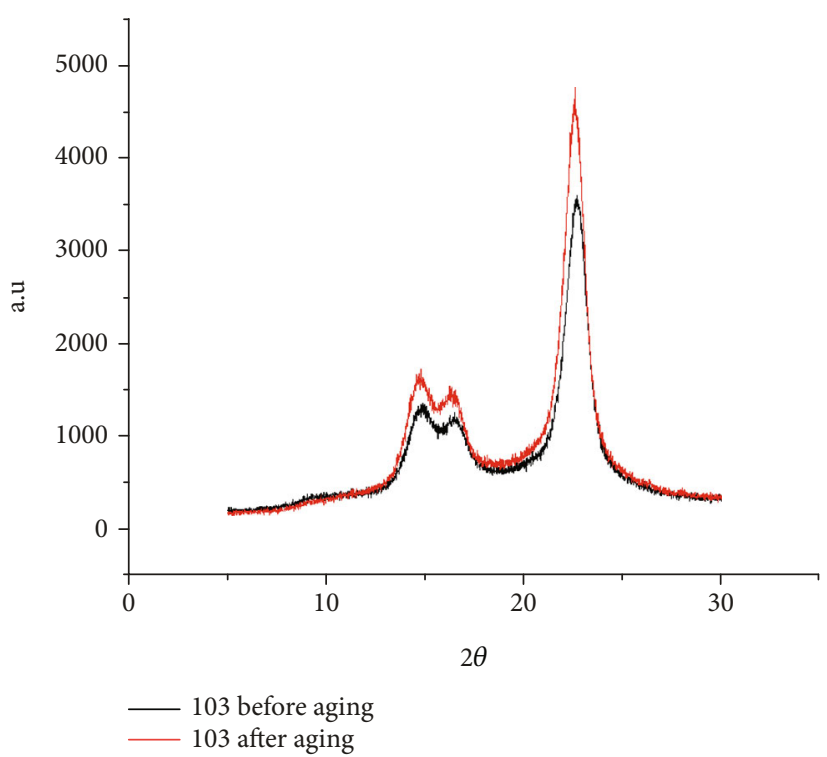

(b)

FIGURE 4: Comparison of the X-ray diffractograms obtained before and after aging for (a) paper sample 1.0.2 (egg white-alum sizing and Hlevan adhesive) and (b) paper sample 1.0.3 (egg white-alum sizing and starch adhesive).

TABle 3: Eigenvalues of factors in PCA.

\begin{tabular}{lcccccccc}
\hline & F1 & F2 & F3 & F4 & F5 & F6 & F7 & F8 \\
\hline Eigenvalue & 4.065 & 2.255 & 1.188 & 0.219 & 0.161 & 0.082 & 0.023 & 0.007 \\
Variability (\%) & 50.818 & 28.190 & 14.844 & 2.741 & 2.012 & 1.023 & 0.285 & 0.087 \\
Cumulative (\%) & 50.818 & 79.008 & 93.852 & 96.593 & 98.605 & 99.628 & 99.913 & 100.000 \\
\hline
\end{tabular}

the other samples. It is known that the reason of microbial attacks on paper is the low $\mathrm{pH}$ condition and yellowing is also the consequence of some microbial products. Remarkable chromatic alteration and lower $\mathrm{pH}$ values were observed for the samples with iron gall ink and different concentrations of Hlevan (1.0.2 and 1.0.3) due to the possible resulting chromophores of the oxidation process during aging [44].

3.3. Crystallinity Index. The flexibility of paper comes from its amorphous regions which can keep moisture in, unlike highly packed crystalline regions. One can conclude that an increase in crystallinity inevitably will result in brittleness [33]. Therefore, the measurement of the crystallinity index for paper can be used as a way of indicating the effect of treatments on cellulose chains. The crystallinity index of samples before and after thermal aging has been presented in Table 2. The crystallinity index did not change drastically, but there is a slight increase for almost all samples. We can conclude that the overall change is a natural result of the thermal aging process.

For samples sized with egg white and alum, Hlevan acted as a plasticizer and preserved the amorphous regions from losing their moisture and becoming more crystalline. What is more, in Figure 4, we can see the obtained diffractograms for these samples and starch-applied samples (1.0.3) before and after aging. The important peaks of the diffractograms are (101), (10i), and (002) reflexes at $15.0^{\circ}, 17.0^{\circ}$, and $22.7^{\circ}$

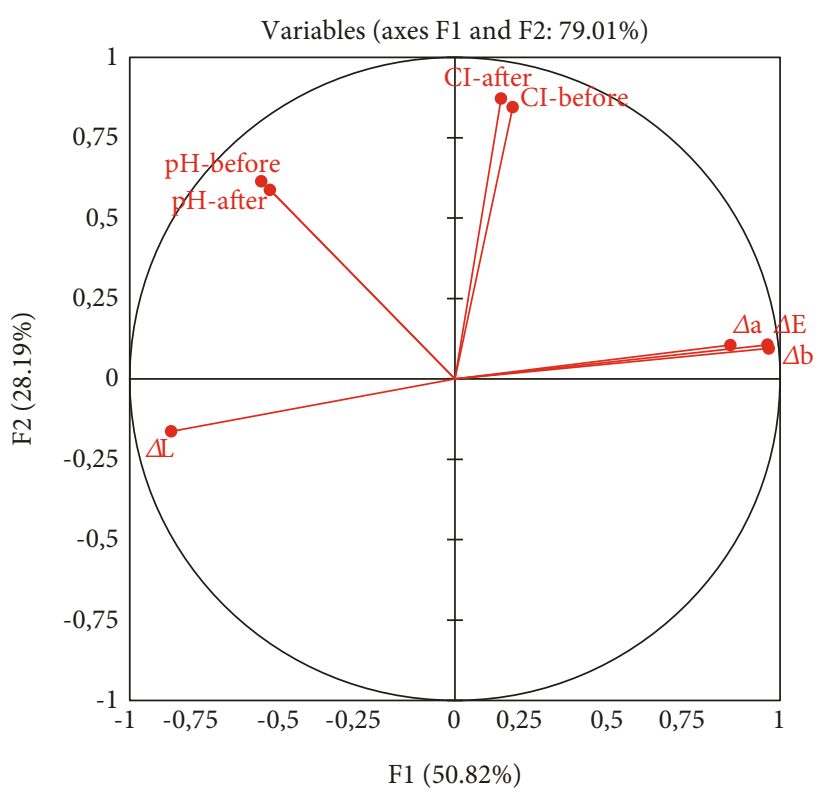

- Active variables

FIGURE 5: The projections of variables over the directions.

which can be related to crystalline cellulose. There is an intensity difference between peaks of the samples 1.0.3 and 1.0.2 at mentioned reflexes. This explains the crystallinity 


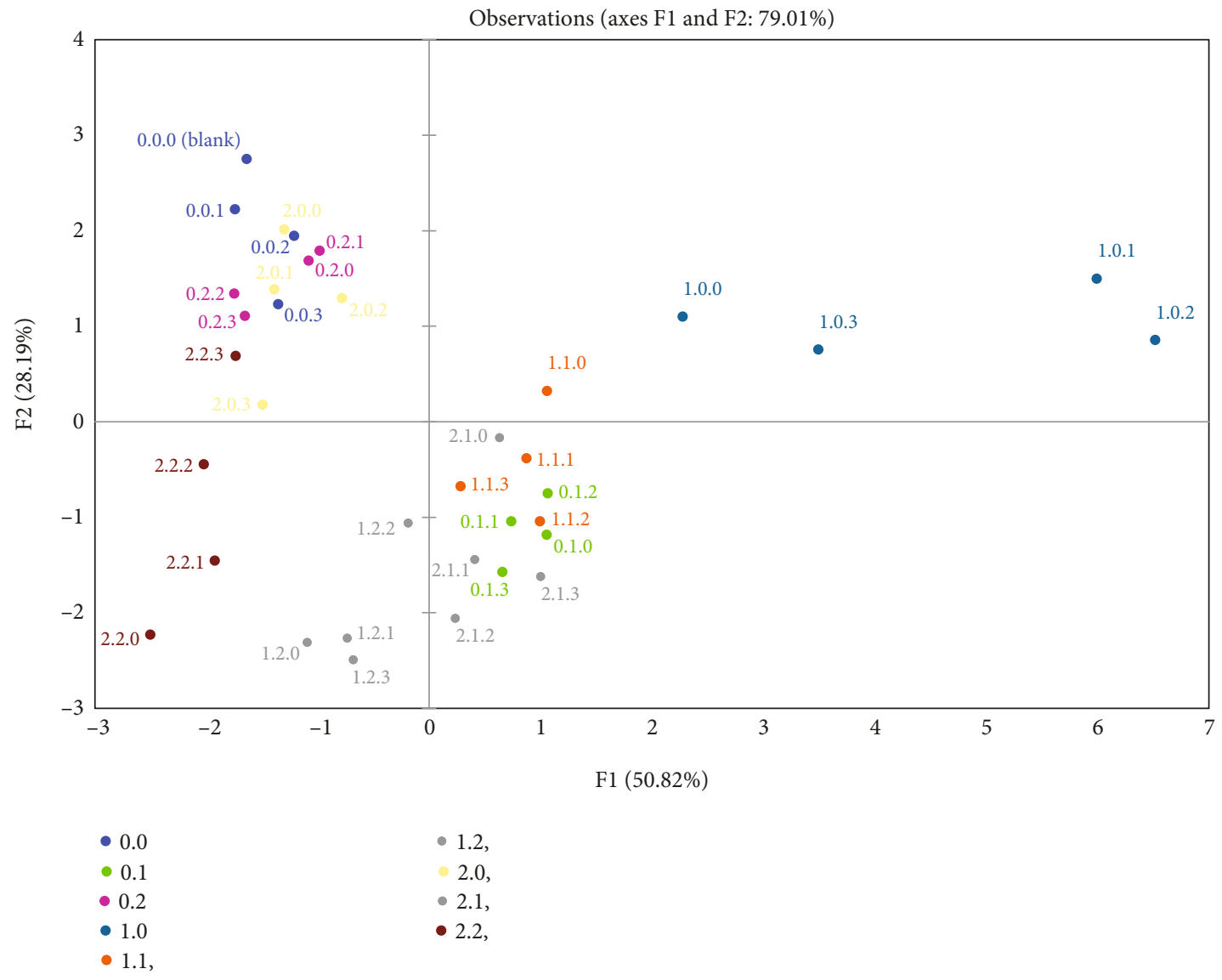

Figure 6: The projection of samples onto the space spanned by the first two principal components (explaining more than $79.01 \%$ of the original variance).

index differences between starch and Hlevan-applied samples after aging.

The XRD results showed that for the samples treated with starch as sizing and carbon black as an ink (i.e., the group of samples 2.2), there were noticeable differences in the amorphous areas but not for the one on which starch was also applied as adhesive (i.e., 2.2.3). When compared with other groups, there is a slight increase in crystallinity after aging which implies that the elasticity decreases for Hlevanapplied samples.

3.4. Principal Component Analysis. In order to differentiate the interaction of differently treated paper samples with Hlevan and starch as adhesives, PCA of all samples were performed. We wanted to see if there is a noticeable effect of treatments on adhesive-applied papers and which one would dominate among selected variables. The two-factor solution was carried out using XLSTAT version 2019.4.2. The first two factors explain over $79.01 \%$ of the total variability in the data which have eigenvalues over 1.00 (see Table 3).

The projections of active variables over the direction determined by F1 are strongly correlated with the chromatic changes, mainly delta $E$ and delta $b$ where the second direction F2 accounts for the differences in crystallinity of the samples (see Figure 5). Inspection of this plot has evidenced that the biggest variability in the dataset comes from color change which is compatible with the findings of yellowing and fading after thermal aging following adhesive applications on paper samples. The first principal component (F1) which holds $50.82 \%$ of the total variability was minorly affected by $\mathrm{pH}$ and crystallinity results.

If we focus on the score plot of observations onto the space spanned by the principal components (Figure 6), clustering between some of the different groups can be observed. Groups 1.1, 2.1, and 0.1 behaved similarly and differentiated from other clusters of comparable groups, i.e., $0.2,0.0$, and 2.0. The differences between these samples involve the second principal component (F2) which accounts for $28.19 \%$ total variability of the samples. Group of 1.0 was separated and partially superpositioned among others which may indicate the importance of sizing material on the behavior of samples. Samples 1.0.1 and 1.0.2 (with $7 \%$ and $10 \%$ Hlevan formulations) were differentiated from 1.0.0 (control) and 1.0.3 (10\% starch). In addition, the samples with iron gall ink clustered together. This suggests that the choice of ink also directed the behavior of samples more than adhesives did upon aging.

\section{Conclusions}

From the results obtained in this study, one may conclude that, although Hlevan caused detectable variations in paper 
samples as much as the level of starch did, other media elements like sizing and ink on paper supports dominated the change in samples in general. Yet, it was obvious that the sizing material affected the aging behavior of Hlevan. Egg white-alum sizing resulted in a more pronounced difference in yellowing for all samples, but the color change was more noticeable for the ones on which egg white-alum sizing and Hlevan were applied together. This may be due to the Maillard reaction that is accelerated in the presence of higher temperature during the aging procedure. Hence, for future studies, moist-heat aging with conditions of $80^{\circ} \mathrm{C}$ and $65 \%$ $\mathrm{RH}$ for 10 days is planned to be operated to examine the effect of both temperature and humidity. In this study, we also concluded that the Hlevan preserved the amorphous regions of fibers for the samples sized with egg white-alum and presented more alkaline nature than starch after aging. The methodology of this study differs from others as the influence of parameters like sizing and ink was also investigated simultaneously. Resulting data were analyzed by PCA to see the contribution of these additional parameters to the adhesive effect on cellulosic substrates. Although chromatic aging characteristics of levan for the egg-white sizing was more definite than the rest, we believe that other excellent and promising chemical properties will enable it to be distinguished from starch and can be improved in future studies.

\section{Data Availability}

The data used to support the findings of this study are included within the article and supplementary information file and can be also available from the corresponding author upon request.

\section{Conflicts of Interest}

The authors declare that there is no conflict of interest.

\section{Acknowledgments}

The authors thank Onur Atak (Marmara University, Department of Textile Engineering) for his help in making color measurements and Fatma Aslanoglu Erbug (Project Conservator at University of College London) for assistance in sizing applications. We gratefully acknowledge the Scientific and Technological Research Council of Turkey (TUBITAK) through Project 116M838.

\section{Supplementary Materials}

In this document list of samples, sample codes and group codes were given in detail. (Supplementary Materials)

\section{References}

[1] E. T. Öner, L. Hernández, and J. Combie, "Review of levan polysaccharide: from a century of past experiences to future prospects," Biotechnology Advances, vol. 34, no. 5, pp. 827844, 2016.
[2] J. Feng, Y. Gu, Y. Quan et al., "Recruiting a new strategy to improve levan production in Bacillus amyloliquefaciens," Scientific Reports, vol. 5, no. 1, p. 13814, 2015.

[3] Y. Gu, J. Zheng, J. Feng et al., "Improvement of levan production in Bacillus amyloliquefaciens through metabolic optimization of regulatory elements," Applied Microbiology and Biotechnology, vol. 101, no. 10, pp. 4163-4174, 2017.

[4] F. Küçükaşik, H. Kazak, D. Güney et al., "Molasses as fermentation substrate for levan production by Halomonas sp.", Applied Microbiology and Biotechnology, vol. 89, no. 6, pp. 1729-1740, 2011.

[5] H. K. Sarilmiser, O. Ates, G. Ozdemir, K. Y. Arga, and E. T. Oner, "Effective stimulating factors for microbial levan production by Halomonas smyrnensis AAD6 ${ }^{\mathrm{T}}$," Journal of Bioscience and Bioengineering, vol. 119, no. 4, pp. 455-463, 2015.

[6] A. Poli, H. Kazak, B. Gürleyendağ et al., "High level synthesis of levan by a novel_Halomonas_species growing on defined media," Carbohydrate Polymers, vol. 78, no. 4, pp. 651-657, 2009.

[7] H. K. Sarilmiser and E. T. Oner, "Investigation of anti-cancer activity of linear and aldehyde-activated levan from Halomonas smyrnensis $\mathrm{AAD6}^{\mathrm{T}}$," Biochemical Engineering Journal, vol. 92, pp. 28-34, 2014.

[8] M. Erginer, A. Akcay, B. Coskunkan et al., "Sulfated levan from Halomonas smyrnensis as a bioactive, heparin-mimetic glycan for cardiac tissue engineering applications," Carbohydrate Polymers, vol. 149, pp. 289-296, 2016.

[9] S. Sam, F. Kucukasik, O. Yenigun, B. Nicolaus, E. T. Oner, and M. A. Yukselen, "Flocculating performances of exopolysaccharides produced by a halophilic bacterial strain cultivated on agro-industrial waste," Bioresource Technology, vol. 102, no. 2, pp. 1788-1794, 2011.

[10] A. D. Sezer, H. K. Sarılmışer, E. Rayaman, A. Çevikbaş, E. T. Öner, and J. Akbuğa, "Development and characterization of vancomycin-loaded levan-based microparticular system for drug delivery," Pharmaceutical Development and Technology, vol. 22, no. 5, pp. 627-634, 2016.

[11] A. D. Sezer, H. Kazak, E. T. Öner, and J. Akbuğa, "Levan-based nanocarrier system for peptide and protein drug delivery: optimization and influence of experimental parameters on the nanoparticle characteristics," Carbohydrate Polymers, vol. 84, no. 1, pp. 358-363, 2011.

[12] C. Costa, A. S. Henriques, C. Pires et al., "The dual role of Candida glabrata drug: $\mathrm{H}^{+}$antiporter CgAqr1 (ORF CAGLOJ09944g) in antifungal drug and acetic acid resistance," Frontiers in Microbiology, vol. 4, p. 170, 2013.

[13] M. S. Bostan, E. C. Mutlu, K. Hande, S. Sinan Keskin, E. T. Oner, and M. S. Eroglu, "Comprehensive characterization of chitosan/PEO/levan ternary blend films," Carbohydrate Polymers, vol. 102, pp. 993-1000, 2014.

[14] T. D. Gomes, S. G. Caridade, M. P. Sousa et al., "Adhesive freestanding multilayer films containing sulfated levan for biomedical applications," Acta Biomaterialia, vol. 69, pp. 183195, 2018.

[15] A. Osman, E. T. Oner, and M. S. Eroglu, "Novel levan and pNIPA temperature sensitive hydrogels for 5-ASA controlled release," Carbohydrate Polymers, vol. 165, pp. 61-70, 2017.

[16] J. Combie, A. Haag, P. Suci, and G. Geesey, "Adhesive produced by microorganisms," in Agricultural Applications in Green Chemistry, ACS Symposium Series, W. M. Nelson, Ed., pp. 53-62, American Chemical Society, WA, USA, 2004. 
[17] I. Alexopoulou and S. Zervos, "Paper conservation methods: an international survey," Journal of Cultural Heritage, vol. 21, pp. 922-930, 2016.

[18] S. Zervos and A. Moropoulou, "Methodology and criteria for the evaluation of paper conservation interventions: a literature review," Restaurator, vol. 27, no. 4, pp. 219-274, 2006.

[19] I. da Silva Borges, M. H. Casimiro, M. F. Macedo, and S. O. Sequeira, "Adhesives used in paper conservation: chemical stability and fungal bioreceptivity," Journal of Cultural Heritage, vol. 34, pp. 53-60, 2018.

[20] J. Combie and E. T. Öner, "From healing wounds to resorbable electronics, levan can fill bioadhesive roles in scores of markets," Bioinspiration \& Biomimetics, vol. 14, no. 1, article 011001, 2019.

[21] M. Barkeshli, "Historical and scientific analysis on sizing materials used in Iranian manuscripts and miniature paintings," in The Book and Paper Group Annual (2003), vol. 22, pp. 9-16, the Book and Paper Group (BPG), 2003.

[22] J. Kosek and C. Barry, "Investigating the condition of iron gall ink drawings: developing an assessment survey," Journal of the Institute of Conservation, vol. 42, no. 3, pp. 191-209, 2019.

[23] S. Zervos and I. Alexopoulou, "Paper conservation methods: a literature review," Cellulose, vol. 22, no. 5, pp. 2859-2897, 2015.

[24] A. Syrafi, "Golzari safa," in Ketab-Arayi Dar TamadduniEslami, N. Mayel-Heravi, Ed., pp. 239-255, Islamic Research Centre of Astan-e Qods-eRazavi, Mashhad, 1543.

[25] H. A. Rostamdari, "Khat va morakkab," in Ketab-Arayidar Tamaddon-e Eslami, N. Mayel-Heravi, Ed., pp. 323-342, Islamic Research Centre of Astan-e Qods-e Razavi, Mashhad, 1571.

[26] M. Rifat, Golzari Savab, Nefes Zade Ibrahim Tashih, Fine Arts Academy Publication, Turkey, Istanbul, 1938.

[27] S. Thoma, "Making and testing iron gall ink," West Dean College Books and Library MaterialsMarch 2015. https://www .westdean.org.uk/study/school-of-conservation/blog/booksand-library-materials/making-testing-iron-gall-ink.

[28] C. A. Mitchell, Inks: their composition and manufacture, including method sof examination and a full list of British patents, C. Griffin \& Co, London, UK, 1937.

[29] B. A. Erkorkmaz, O. Kirtel, Ö. A. Duru, and E. T. Öner, "Development of a cost-effective production process for Halomonas levan," Bioprocess and Biosystems Engineering, vol. 41, no. 9, pp. 1247-1259, 2018.

[30] C. R. Y. S. T. A. L. Maitland, "Microscopy for paper conservation: comparing various adhesives and examining wheat starch paste preparation methods," Poster presented at AIC's 38th Annual Meeting, Milwaukee, USA, 2010.

[31] H. E. Ahmed and F. N. Kolisis, "An investigation into the removal of starch paste adhesives from historical textiles by using the enzyme $\alpha$-amylase," Journal of Cultural Heritage, vol. 12, no. 2, pp. 169-179, 2011.

[32] S. Manente, A. Micheluz, R. Ganzerla, G. Ravagnan, and A. Gambaro, "Chemical and biological characterization of paper: a case study using a proposed methodological approach," International Biodeterioration \& Biodegradation, vol. 74, pp. 99-108, 2012.

[33] K. Ward Jr., "Crystallinity of cellulose and its significance for the fiber properties.," Textile Research Journal, vol. 20, no. 6, pp. 363-372, 2016.
[34] L. Segal, J. J. Creely, A. E. Martin, and C. M. Conrad, “An empirical method for estimating the degree of crystallinity of native cellulose using the X-ray diffractometer," Textile Research Journal, vol. 29, no. 10, pp. 786-794, 2016.

[35] Y. Cao and H. Tan, "Study on crystal structures of enzymehydrolyzed cellulosic materials by X-ray diffraction," Enzyme and Microbial Technology, vol. 36, no. 2-3, pp. 314-317, 2005.

[36] Y. Wang, Y. Zhao, and Y. Deng, "Effect of enzymatic treatment on cotton fiber dissolution in $\mathrm{NaOH} /$ urea solution at cold temperature," Carbohydrate Polymers, vol. 72, no. 1, pp. 178-184, 2008.

[37] H. E. Ahmed, "Protease enzyme used for artificial ageing on modern cotton fabric for historic textile preservation and restoration," International Journal of Conservation Science, vol. 4, no. 2, 2013.

[38] J. M. Ames, "The Maillard reaction," in Biochemistry of Food Proteins, pp. 99-153, Springer, Boston, MA, 1992.

[39] L. C. Maillard, "Action des acides amines sur les sucres; formation de melanoidines par voie méthodique," Comptes rendus de l'Académie des Sciences, vol. 154, pp. 66-68, 1912.

[40] V. Bukovsksy, "Yellowing of newspaper after deacidification with methyl magnesium carbonate," Restaurator, vol. 18, pp. 25-38, 1997.

[41] W. Yanjuan, F. Yanxiong, W. Tan, and L. Chunying, "Preservation of aged paper using borax in alcohols and the supercritical carbon dioxide system," Journal of Cultural Heritage, vol. 14, no. 1, pp. 16-22, 2013.

[42] T. Trafela, M. Strlic ${ }^{2}$ J. Kolar et al., "Nondestructive analysis and dating of historical paper based on IR spectroscopy and chemometric data evaluation," Analytical Chemistry, vol. 79, no. 16, pp. 6319-6323, 2007.

[43] R. R. A. Hassan, "Behavior of archaeological paper after cleaning by organic solvents under heat accelerated aging," Mediterranean Archaeology and Archaeometry, vol. 15, no. 3, pp. 141-150, 2015.

[44] A.-L. Dupont, "Study of the degradation of gelatin in paper upon aging using aqueous size-exclusion chromatography," Journal of Chromatography A, vol. 950, no. 1-2, pp. 113-124, 2002. 